\title{
Pharmacokinetic interaction between alcohol and two of the commonly prescribed anti-hypertensive drugs-amlodipine and nebivolol
}

\author{
Geethalakshmi Gunasekaran*, Ruckmani A., Arjun Arumugam Olaganathan, Arun Kumar R.
}

Department of Pharmacology, Chettinad Hospital and

Reasearch Centre,

Kanchipuram, Tamil Nadu, India

Received: 06 March 2017

Accepted: 14 March 2017

*Correspondence to:

Dr. Geethalakshmi

Gunasekaran,

Email: geeth5n@gmail.com

Copyright: (C) the author(s), publisher and licensee Medip Academy. This is an openaccess article distributed under the terms of the Creative Commons Attribution NonCommercial License, which permits unrestricted noncommercial use, distribution, and reproduction in any medium, provided the original work is properly cited.

\begin{abstract}
Background: Alcoholism is one of the leading causes of secondary hypertension. Many a times in clinical setting, many hypertensive patients also happen to be alcoholics, either social/moderate drinkers or suffer from alcohol dependence. For these patients, cessation of alcohol consumption is advocated and counselled as part of diet and lifestyle modifications. However, compliance rates have been found to be variable. Therefore, these patients consume alcohol and also take anti-hypertensive medications on a day-to-day basis. This study was initiated to explore influence of alcohol on the pharmacokinetics of antihypertensive drugs - Amlodipine and Nebivolol.

Methods: 24 human volunteers were recruited for the study after obtaining informed consent. 12 volunteers each for Amlodipine, Nebivolol and alcohol's effect on the drugs respectively were evaluated. Two standard drinks of alcohol were administered in respective period as per randomization. Clinical confinement and blood sampling was carried out as per ethics committee approved protocol in accordance with good clinical practice principles.

Plasma samples were analyzed using validated LC-MS/MS bio-analytical method, for quantification of Nebivolol and Amlodipine in lines with good laboratory practice principles. Pharmacokinetic and statistical analysis of results was evaluated using WinNonlin Version 5.3.

Results: The pharmacokinetic parameters were evaluated statistically and ANOVA results suggest that despite changes in individual parameters, the bioavailability was comparable, as both $\mathrm{Cmax}$ and AUC0-inf were well within the range of $80-125 \%$.

Conclusions: It is clearly observed that there are variations in all pharmacokinetic parameters when the drug is administered with alcohol. However, the same are well within acceptable limits and bioavailability of the drugs is comparable when administered with alcohol. Hence, two standard drinks of alcohol have limited effect on the pharmacokinetics of Amlodipine and Nebivolol. Further studies are required to evaluate influence of higher and frequent doses of alcohol on pharmacokinetics of Amlodipine and Nebivolol.
\end{abstract}

Keywords: Alcohol, Anti-hypertensives, Bioavailbility, Pharmacokinetics

\section{INTRODUCTION}

Alcohol, by far, is the most widely and frequently used and abused agent. It is a psychoactive substance with dependence-producing properties that has been widely used in many cultures for centuries. Alcoholism is one of the major causes of socio-econominc burden in India. ${ }^{1}$ the pattern of consumption of alcohol over the years clearly indicates a rise in the drinking habits among Indians. Most popularly consumed are spirits containing 40-60\% alcocol by volume. ${ }^{2}$ Hypertension is a global public health issue. ${ }^{3}$ Globally, cardiovascular disease accounts for $\sim 17$ million deaths a year, nearly one third of the total. $^{4}$ Of these, complications of hypertension account for 9.4 million deaths worldwide every year at least $45 \%$ of deaths due to heart disease and $51 \%$ of deaths due to cerebrovascular events. ${ }^{4,5}$

In India, hypertension is a major health concern and its prevalence is rapidly increasing among both urban and rural populations. 6,7 
A survey of 26,000 adults in South India showed a hypertension prevalence of $20 \%$ (men $23 \%$ and women $17 \%$ ) but $67 \%$ of those with hypertension were unaware of their diagnosis. ${ }^{8}$

Populous studies have been conducted exploring into the secondary causes of hypertension including alcohol induced hypertension and the established need for lifestyle modifications and reduction/cessation of alcohol consumption for management of hypetension. Most of these studies were conducted in hypertensive patients to better understand the etiology, drug interactions and to draw practical treatment goals and guidelines.

However, interaction study between anti-hypertensive drugs and alcohol in humans are deficient. The studies conducted have excluded alcoholic participants and in fact, require the study participants to abstain from alcohol atleast two weeks prior to study enrollment and during the period of the study to avoid interference in the results. ${ }^{9}$

Thus, many a times in a clinical setting, many hypertensives also happen to be alcoholics, either social/moderate drinkers or those who suffer from alcohol dependance. ${ }^{10}$ Therefore, these patients consume alcohol and also take anti-hypertensive medications on a day to day basis.

However, the clinical trials studying the pharmacokinetics of the anti-hypertensive drugs are chiefly conducted in healthy individuals and/or requires abstinence from alcohol at least 24 hours before each study period. ${ }^{10}$ But not only in antihypertensive drug trials, in most of the clinical trials alcoholics are excluded from recruitment.

Consequently, this study was premediated to explore the influence of alcohol on the pharmcokinetics of antihypetensive drugs.

\section{Objectives}

Primarily, to study the influence of ethanol on the pharmacokinetic parameters of two commonly prescribed anti-hypertensive drugs, Amlodipine and Nebivolol, in adult male population following oral administration of the medications under fasting conditions. Secondary objective was to ensure safety and wellbeing of the study participants during the course of the study.

\section{METHODS}

The protocol and informed consent document were reviewed and approved by Institutional Human Ethics Committee of Chettinad Hospital and Research Institute on 12-MAY-2015.

The study was conducted as per ICMR Ethical Guidelines for Biomedical Research on Human Subjects (2006), ICH
GCP, Schedule Y, 'Good Clinical Practices for Clinical Research in India' Guidelines, and Declaration of Helsinki in Azidus Laboratories Ltd.

The study was as an open label, two treatment, two periods, two sequences, single dose, and cross over, oral pharmacokinetic study in 24 male subjects with history of moderate alcohol consumption under fasting conditions with 11 days washout between periods.

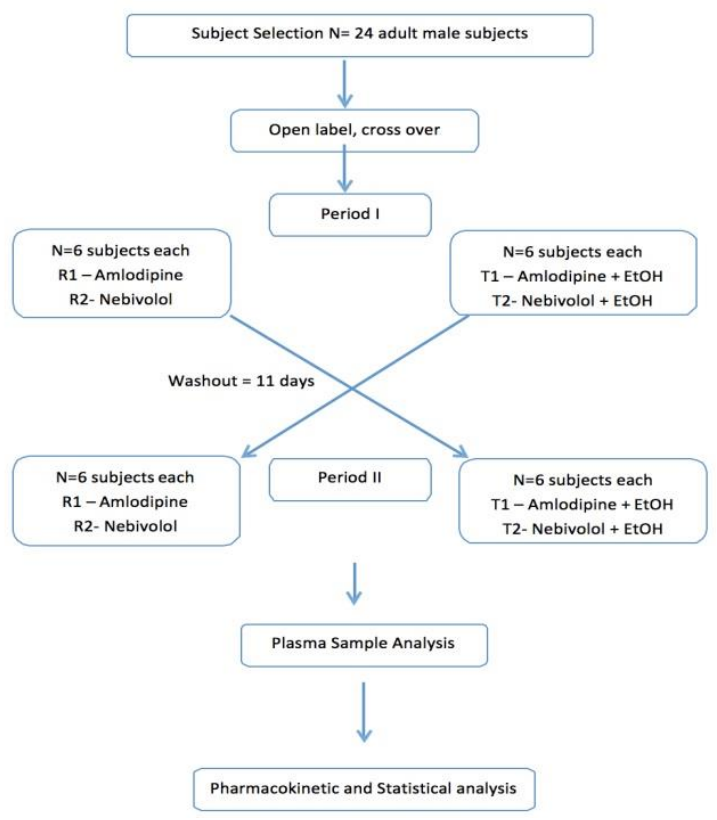

Figure 1: Study design.

Pharmacokinetic interaction was determined by statistical comparison of log-transformed data of AUC0-t, AUC0 and Cmax for the test and reference products.

Written informed consent was obtained from each volunteer after providing ample amount of time to read the informed consent document, followed by oral presentation by Investigator. All subjects underwent a screening procedure prior to study-demography, medical, personal history, general and systemic examination, vital parameters measurement, and laboratory investigationsECG, hematological, biochemical, serological and urinary analysis. The subjects were selected based on the following:

\section{Inclusion criteria}

Volunteers meeting all the following criteria were considered for enrollment:

- Healthy male volunteers of 20 to 45 years

- Willing to give informed written consent and complies with study requirements.

- $\quad$ BMI 18.50-24.99Kg/m2 and weight $>50 \mathrm{~kg}$

- Healthy individual evaluated by personal history, medical history and general clinical examination. 
- History of moderate alcohol consumption, 2-3 standard drinks per day and not exceeding 12-14 drinks per week.

- Vital parameters: Within normal acceptable range

- Normal bio-chemical, hematological and urinary parameters, Chest $\mathrm{X}$ - ray PA view and 12 leads ECG

- Negative for HIV 1 and 2, Hepatitis B, Hepatitis C, and Syphilis tests

- $\quad$ Negative alcohol breath analysis (performed on the day of check in of both periods).

\section{Exclusion criteria}

Subjects meeting any of these criteria were not considered for study

- $\quad$ Surgery, Systemic NCD, electrolyte imbalances and hypo-volemia

- Clinical history suggestive of cardiac, gastrointestinal, respiratory, hepatic, renal, endocrine, neurological, metabolic, psychiatric, hematological systems or glaucoma judged to be clinically significant

- Dysphagia and any medical disorder that is of significance in the investigator's opinion

- Smoking 9 or more cigarettes/beedies per day and/or inability to withhold smoking or consumption of tobacco containing products during the study

- Hypersensitivity to any related drugs or excipients in the formulation

- Intake of drugs which potentially modify kinetics/ dynamics study medications judged to be clinically significant by the investigator

- Subject who had participated in a clinical trial/donated blood in the past 3 months / drug abuse in past 12 months

- Habituation to coffee, tea or other xanthine containing products

Withdrawal of subjects from therapy or assessment

- $\quad$ Subject withdraws consent

- Development of intolerable adverse event

- $\quad$ Subjects who experience emesis at any time during the study and/or significant diarrhea, in any period

- Development of inter-current illness for which the subject requires drugs that interact with study medications

- Violation of the protocol or occurrence of a significant protocol violation during study

- The investigator feels that in the best interest of the subject's health, the subject is to be withdrawn

- Data not known before starting the trial become available and raise concern about the safety of the study drug so that continuation would pose potential risk to any particular subject

- Subject is non-cooperative and/or un-disciplined
The eligible subjects were enrolled and randomly assigned to one of the sequences of test $(\mathrm{T})$ and reference (R).

Reference drugs were Amlogard 5mg (Pfizer) and Nebicip 5mg (Cipla). The Test drugs were the same along with administration of ethanol.

Ethanol used - Brandy containing $40 \%$ alcohol by volume. 2 standard drinks of $30 \mathrm{ml}$ each (total $60 \mathrm{~mL}$ ) were administered to subjects to elicit any influence of alcohol on pharmacokinetics.

Table 1: Investigational products.

\begin{tabular}{|ll|}
\hline Reference & Test \\
\hline Amlodipine (R1) & Amlodipine + Ethanol (T1) \\
\hline Nebivolol (R2) & Nebivolol + Ethanol (T2) \\
\hline
\end{tabular}

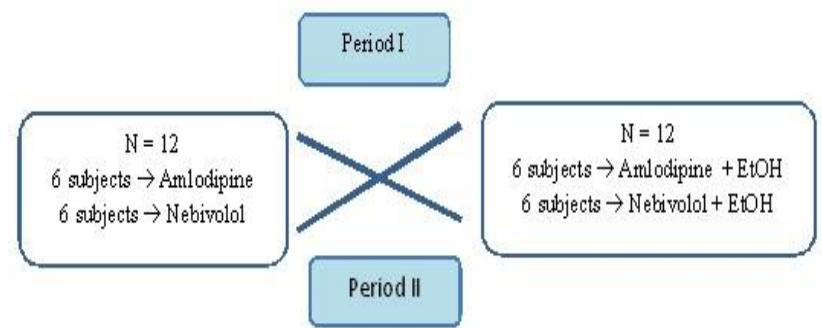

Figure 2: Randomization.

Selected subjects were housed in clinical facility from minimum 12 hours prior to drug administration and until 24.00 hours post dose in each period. After check-in, subjects received standard dinner and fasted overnight for at least 10 hours. The subjects received standard food at 04.00, 08.00, $12.00 \mathrm{~h}$ post-dose. Subjects were not provided coffee, tea or any other xanthine containing food or drinks and carbonated drinks. They were instructed not to consume juice/food containing citrus fruits during entire duration of study.

A single oral dose of investigational drug was given to subjects with water at ambient temperature at fixed time points in sitting posture, in each period, followed by mouth check to assess the compliance to dosing. Alcohol was given post dosing and the subjects were advised to consume it over $30 \mathrm{~min}$. Subjects had to be seated for $4 \mathrm{~h}$ after dosing. No exercise or strenuous physical activities were permitted. Blood samples were collected at 00.00 hour (pre-dose), 00.50, 01.00, 02.00, 03.00, 04.00, 05.00, 06.00, 07.00, 08.00, 09.00, 10.00, 11.00, 12.00, 13.00, $14.00,16.00,20.00,24.00,48.00$ and $72.00 \mathrm{~h}$ post dose (21 samples of $2 \mathrm{~mL}$ each). First 19 samples were collected as in house through an indwelling cannula placed in a forearm/arm vein and remaining through direct venous puncture using K3EDTA pre-labeled vacutainers as ambulatory. Total blood loss was 119.0 $\mathrm{mL}$. The samples were centrifuged at $4000 \mathrm{rpm}$ for 10 
minutes at $4 \pm 2{ }^{\circ} \mathrm{C}$; separated into two aliquots and stored at $-70{ }^{\circ} \mathrm{C}$ till analysis.

Subjects were continuously monitored and wellbeing was investigated by physical examination and vital signs measurements at check-in, 00.00, 02.00, 04.00, 12.00, 24.00 hours. There were no events of adverse effects or deviations in the study.

\section{Bio-analytical methodology}

Estimation of Amlodipine and Nebivolol in plasma was done using validated Liquid Chromatography Mass spectometry (LC-MS/MS) bioanalytical method. Method validation included the following parameters:

- $\quad$ Specificity- Specificity was performed on at least six independent sources of the same matrix.

- Sensitivity- Sensitivity was performed at LLOQ concentration.

- Precision and Accuracy- Three precision and accuracy batches were checked. The between batch precision for low, medium and high QC samples should be $\leq 15 \%$, whereas for LLOQQC $\leq 20 \%$. Between batch mean accuracy should be within $\pm 15 \%$ of nominal value at low, medium and high QC concentrations, whereas for LLOQQC it should be $\pm 20 \%$.

- $\quad$ Stability - Freeze-thaw, bench top, in-injector, long term stability and stock solution stability will be performed.

- Recovery- For analyte and internal standard from biological matrix.

- Dilution Integrity

Samples were segregated and transferred to bio-analytical facility and stored at $-70 \mathrm{oC}$ in an Ultra-Low Temperature Freezer.

\section{Quantification levels}

Amlodipine: $0.152 \mathrm{ng} / \mathrm{mL}$ to $11.496 \mathrm{ng} / \mathrm{mL}$

Nebivolol: $0.057 \mathrm{ng} / \mathrm{mL}$ to $7.860 \mathrm{ng} / \mathrm{mL}$

The analytes were selectively isolated from $100 \mu \mathrm{L}$ Plasma by Liquid-Liquid extraction and estimated using mass spectrometric detection on Symmetry C18 $5 \mu \mathrm{m}$ column.

A batch consisted of aqueous MQC, Reconstitution Solution, K3EDTA human blank plasma (standard blank), K3EDTA human Blank plasma containing IS (standard zero), calibration curve standards, two sets of Quality control samples at LQC, MQC and HQC, interspersed among subject samples. All samples from a subject were analysed in same batch.

\section{Pharmacokinetic and statistical analysis}

The drug concentration data of all 24 completed subjects were included for analysis. Pharmacokinetic parameters were calculated using WinNonlin ${ }^{\circledR}$ (v5.3). The mean, standard deviation, geometric mean, coefficient of variation $(\mathrm{CV} \%)$, minimum, median, maximum and range were calculated for Cmax, AUC0-t,AUC0- $\infty$, Tmax, T1/2, Kel and AUC_\% Extrap_Obs.

\section{Statistical analysis}

Analysis of variance (ANOVA) was performed on the Ln- transformed data of Cmax, AUC0-t and AUC0- $\infty$ using GLM procedure of SAS® (v9.2). The analysis of variance model included sequence, period and treatment as fixed effect and the subjects nested within the sequence as random effect. The sequence effect was tested at 0.10 level of significance and other main effects at 0.05 level of significance against the residual error from ANOVA as the error term. Based on comparisons of test and reference products for Ln-transformed $\mathrm{C}_{\max }$, AUC0-t and AUC0- $\infty$ data, the ratio of the least square mean and $90 \%$ confidence intervals were calculated.

\section{RESULTS}

Data from all 24 subjects were used for the pharmacokinetic and statisitical evaluation. All trial subjects completed the study and there were no adverse events. The Ln- transformed data of Cmax, AUC0-t and AUC0- $\infty$ was analyzed using GLM procedure of SAS® (v 9.2) for Test treatments comparing it to respective Reference treatments. The statistical output results were as follows:

Amlodipine + Alcohol (T1) vs. Amlodipine (R1):

The test/reference ratios obtained for the Cmax, AUC0-t

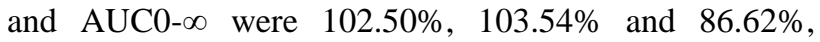
respectively. The $90 \%$ confidence interval calculated for the three pharmacokinetic parameters - Cmax, AUC0-t and $\mathrm{AUC} 0-\infty$ were all well within the bioequivalence limits of $80 \%-125 \%$.

Table 2: $90 \%$ Confidence interval output - pk of nebivolol administered with alcohol (T2) compared with nebivolol pk (R2).

\begin{tabular}{|lllll|}
\hline Dependent & $\begin{array}{l}\text { Ratio } \\
\text { [\%Ref] }\end{array}$ & $\begin{array}{l}\text { CI_90_- } \\
\text { Lower }\end{array}$ & $\begin{array}{l}\text { CI_90_ } \\
\text { Upper }\end{array}$ & $\begin{array}{l}\text { ISC } \\
\text { V }\end{array}$ \\
\hline Ln (Cmax) & 121.34 & 96.99 & 151.81 & 0.309 \\
\hline $\begin{array}{l}\text { Ln } \\
\text { (AUClast) }\end{array}$ & 116.39 & 93.33 & 145.16 & 0.305 \\
\hline $\begin{array}{l}\text { Ln (AUCINF } \\
\text { obs) }\end{array}$ & 105.69 & 84.99 & 131.43 & 0.301 \\
\hline
\end{tabular}

The intra-subject variation observed for these parameters ranges between 9-14\%. The Tmax remained unchanged, 
while the t1/2 was delayed by 2 hours compared to a mean of 20 hours without alcohol.

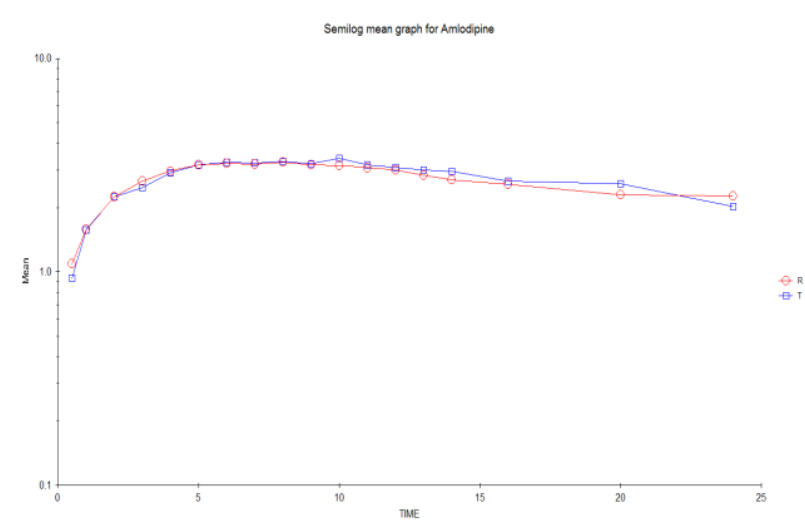

Figure 3: Mean semi-log plot comparing pk of amlodipine administered with Alcohol (T1) with amlodipine pk (R1) against concentration and time.

Nebivolol + Alcohol (T2) vs. Nebivolol (R2):

The ratios of test to reference products obtained were $121.34 \%, 116.39 \%$ and $105.69 \%$ for Cmax, AUC0-t and AUC0- $\infty$, respectively. The calculated $90 \%$ confidence intervals for the parameters were within the acceptable bioequivalence limits.

Table 3: $90 \%$ Confidence interval output - pk of nebivolol administered with alcohol (T2) compared with nebivolol pk (R2).

\begin{tabular}{|lllll|}
\hline Dependent & $\begin{array}{l}\text { Ratio } \\
\text { [\%Ref] }\end{array}$ & $\begin{array}{l}\text { CI_90_ } \\
\text { Lower }\end{array}$ & $\begin{array}{l}\text { CI_90_ } \\
\text { Upper }\end{array}$ & ISCV \\
\hline Ln (Cmax) & 121.34 & 96.99 & 151.81 & 0.309 \\
\hline $\begin{array}{l}\text { Ln } \\
\text { (AUClast) }\end{array}$ & 116.39 & 93.33 & 145.16 & 0.305 \\
\hline $\begin{array}{l}\text { Ln } \\
\text { (AUCINF_o } \\
\text { bs) }\end{array}$ & 105.69 & 84.99 & 131.43 & 0.301 \\
\hline
\end{tabular}

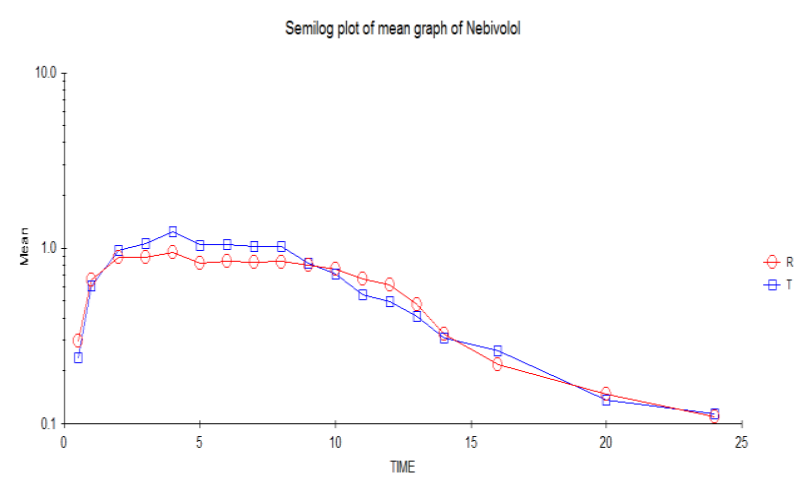

Figure 4: Mean semi-log plot comparing pk of nebivolol administered with alcohol (T2) with nebivolol pk (R2) against concentration and time.
The intra-subject coefficient of variation obatined for Nebivolol was $30 \%$.

A similar trend of unmodified Tmax and prolonged $\mathrm{t} 1 / 2$ was observed with Nebivolol.

\section{DISCUSSION}

\section{Pharmacokinetic parameters}

The objective of the study was to compare the pharmacokinetics of Amlodipine and Nebivolol when it is administered with and without alcohol respectively.

All 24 recruited human volunteers completed the entire course of study and all the subjects' data were included in statistical analysis.

Pharmacokinetic analysis was performed using the WinNonlin ${ }^{\circledR}$ v5.3 for the following pharmacokinetic parameters Cmax, Tmax, AUC0-t, AUC0- $\infty$, T1/2, Kel and AUC\% Extrap_Obs .Analysis of variance (ANOVA) consistent with two one-sided test for bioequivalence, ratio analysis and $90 \% \mathrm{CI}$ for ratio of LSM of Lntransformed data of $\mathrm{Pk}$ parameters for test and reference products were also calculated.

Based on the results obtained, it is clearly observed that there are variations in all pharmacokinetic parameters when the drug is administered along with alcohol.

All the Test groups, which were administered alcohol with the respective drug (Amlodipine and Nebivolol) show variations in bioavailability which is indicated by Cmax and AUC ratios (Table 7 and Table 9).

However, the $90 \%$ CI output comparing the effect of alcohol administration on PK of Amlodipine and Nebivolol also indicate comparable bioavailability, which is reflected in the compared parameters $\left(\mathrm{C}_{\max }, \mathrm{AUC}_{0-\mathrm{t}}\right)$ falling within the recommended limits of $80 \%-125 \%$ for bioequivalence. $^{11}$

Studies have been previously conducted which report little effect of alcohol on the pharmacokinetics of other beta blockers such as acebutolol and the findings of the present study is in alignment with the same. ${ }^{12}$

But it is observed that $90 \%$ CI of $\mathrm{AUC}_{0 \text {-inf }}$ does not fall within the $80 \%-125 \%$ limits, for Amlodipine (Table 2) and for Nebivolol (Table 3). Further investigation is required with a larger sample size to observe changes in $90 \%$ CI of $\mathrm{AUC}_{0 \text {-inf. }}$

The intra subject variability for Cmax and AUC was found to be around $31 \%$ for Nebivolol. A public assessment report reviewed by the European Medicines Agency describes an intrasubject variability of $15 \% 13$ which was conducted in a two way crossover design without administration of alcohol. Various 
bioequivalence study reports on healthy volunteers also suggest a low intra subject variation. ${ }^{14}$ The high intra subject CV observed in the current study is unprecedented and it suggests that administration of alcohol can cause significant changes in the within subject concentration profile of Nebivolol, possibly through interference in drug metabolism. However, the ANOVA output indicates that all parameters were within the range of acceptance, irrespective of the variation observed.

In case of Amlodipine, the literature reported intra subject CV was between $10-14 \%$ for bioequivalence studies in healthy volunteers. ${ }^{15}$ The current study shows an intrasubject $\mathrm{CV}$ of $9-13 \%$ which is similar to the literature reported values. This suggests that alcohol did not influence the within subject variation.

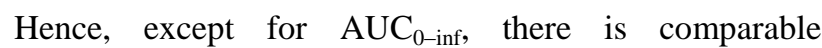
bioavailability observed for both drugs with and without administration of alcohol indicating that the pharmacokinetics are not largely influenced by two standard drinks of alcohol.

Alcohol and drug metabolism: Several researchers have demonstrated the influence of alcohol on drug metabolism, especially through hepatic enzyme interaction. Alcohol is known to induce CYP3A4 enzyme, which is also responsible for metabolizing Amlodipine. ${ }^{16,17}$ Hence, theoretically there could be potential differences in Amlodipine concentration when administered with alcohol, due to increased metabolism which could possibly be the reason for changes observed in the pharmacokinetic parameters. However, these changes are minimal and well within acceptable limits upon statistical evaluation.

On the other hand, Nebivolol is metabolized by CYP2D6 and previous research suggests that alcohol has very little effect on CYP2D6. ${ }^{18}$ However; alcohol can cause potential interference in absorption of many drugs by delaying gastric emptying and in turn influence pharmacokinetics. This could also be a possible reason for the pharmacokinetic variations observed in both Nebivolol and Amlodipine groups.

Interestingly, all the individual parameter results indicate changes after two standard drinks of alcohol but remain within the acceptable range (80\%-125\%) demonstrating comparable bioavailability, in case of both Amlodipine and Nebivolol.

\section{Safety evaluation}

Safety was evaluated throughout the study and there was no adverse event(s). Hence, it can be concluded that both test and reference products are safe and well tolerated at the selected dose when given with alcohol. Post study assessment was performed and no significant deviation was observed compared with pre study values.

\section{Limitations}

The current study only evaluates the influence of two standard drinks of alcohol on the pharmacokinetics of Amlodipine and Nebivolol. Further studies are required to evaluate the effect of higher and more frequent dosing of alcohol on the pharmacokinetics of these drugs.

\section{CONCLUSION}

Based on the results obtained, it can be concluded that two standard drinks of Alcohol have limited influence on pharmacokinetics of Amlodipine and Nebivolol.

Both the drugs have comparable bioavailability when administered with alcohol, based on pharmacokinetics well within acceptable limits. Further studies are required to evaluate the effect of higher doses of alcohol on the pharmacokinetics of both Amlodipine and Nebivolol.

\section{ACKNOWLEDGEMENTS}

Author owes her heartfelt gratitude to Chettinad Hospital and Research Institute and Azidus Laboratories Ltd. for enabling her to take up this research.

Author expresses her deep and sincere gratitude to Dr. A. Ruckmani, Professor and Head, Department of Pharmacology, for being my guide and mentor. Author thanks Dr. R. Arun Kumar, Dr. E. Madhavi, Dr. K. Chandrashekar, Dr. R. Maignanakumar, Dr. S. Saradha, Dr. M. Duraivel, Dr. R. Lakshmipathy Prabhu, Mr. Venugopala Rao Konda, Mr. Vinayak Meti, Dr. Keerthana and Dr. S. Shobita Devi of the Department of Pharmacology, CHRI for their guidance and support.

Funding: No funding sources

Conflict of interest: None declared

Ethical approval: Obtained from Institutional Human Ethics Committee of Chettinad Hospital and Research Institute on 12-MAY-2015

\section{REFERENCES}

1. World Health Organization. A global status report on alcohol and health; Geneva, World Health Organization; 2014.

2. Country profile by World Health Organization. Global status report on alcohol and health. XIII; 2014.

3. World Health Organization. A global brief on Hypertension; Geneva, World Health Organization; 2013.

4. World Health Organization. Causes of death: Data sources and methods. 2008. Available at http://www.who.int/healthinfo/global_burden_diseas e/cod_2008_sources_methods.pdf Accessed Oct 2015.

5. Lim S, Vos T, Flaxman A, Danaei G, Shibuya K, Adair-Rohani H. A comparative risk assessment of 
burden of disease and injury attributable to 67 risk factors and risk factor clusters in 21 regions, 19902010: a systematic analysis for the Global Burden of Disease Study 2010. The Lancet. 2012;380(9859):2224-60.

6. Gupta R, Guptha S. Strategies for initial management of hypertension. Indian Journal of Medical Research. 2010;132(5):531-42.

7. Gupta R, al-Odat NA, Gupta VP. Hypertension epidemiology in India: meta- analysis of 50 years prevalence rates and blood pressure trends. Journal of Human Hypertension. 1996;10(7):465-72.

8. Mohan V. e-Prevalence, awareness and control of hypertension in Chennai. The Chennai Urban Rural Epidemiology Study (CURES-52); 2015.

9. Madhukumar S, Gaikwad V. An Epidemiological Study of Hypertension and its risk factors in rural Population of Bangalore Rural District. AJMS. 2012;5(3):264-70.

10. Guidance for Industry Bioavailability and Bioequivalence Studies Submitted in NDAs or INDsGeneral Considerations; 2014.

11. U.S. Department of Health and Human Services. Food and Drug Administration. Center for Drug. Evaluation and Research (CDER). 2001.

12. Zaman R, Wilkins M, Kendall M, Jack D. The effect of food and alcohol on the pharmacokinetics of acebutolol and its metabolite, diacetolol. Biopharm Drug Dispos. 1984;5(1):91-5.

13. MHRA. Public assessment report Nebivolol $5 \mathrm{mg}$ tablets. http://www.mhra.gov.uk/home/groups/par/documents /websiteresources/con033570.pdf Accessed Oct 2015.

14. EMA Public assessment report. Nebivolol Scientific discussion. Available at http://mri.ctsmrp.eu/download/DK_H_1429_001_PAR_1of3.pdf Accessed Oct

15. USFDA Approved drug products. Amlodipine besylate. Available at http://www.accessdata.fda.gov/scripts/cder/daf/index. cfm?event=BasicSearch.process Accessed Oct 2015

16. Kumar S, Earla R, Jin M, Mitra A, Kumar A. Effect of ethanol on spectral binding, inhibition, and activity of CYP3A4 with an antiretroviral drug nelfinavir. Biochemical and Biophysical Research Communications. 2010;402(1):163-67.

17. Zhu Y, Wang F, Li Q, Zhu M, Du A, Tang W. Amlodipine Metabolism in Human Liver Microsomes and Roles of CYP3A4/5 in the Dihydropyridine Dehydrogenation. Drug Metabolism and Disposition. 2013;42(2):245-9.

18. Vincent-Viry M. The Effects of Drinking and Smoking on the CYP2D6 Metabolic Capacity. ASPET. 2000;28(6).

Cite this article as: Gunasekaran G, Ruckmani A, Olaganathan AA, Kumar AR. Pharmacokinetic interaction between alcohol and two of the commonly prescribed anti-hypertensive drugsamlodipine and nebivolol. Int J Basic Clin Pharmacol 2017;6:795-801. 